\title{
ELECTRICAL PROPERTIES OF CUBIC InN AND GaN EPITAXIAL LAYERS AS A FUNCTION OF TEMPERATURE
}

\author{
J.R.L. Fernandez, , V.A. Chitta ${ }^{* *}$, E. Abramof ${ }^{* * *}$, A. Ferreira da Silva ${ }^{* * *}$, J.R. Leite*, \\ A. Tabata*, D.J. As ${ }^{* * * *}$, T. Frey, D. Schikora, and K. Lischka \\ *Instituto de Física da USP, C.P. 66318, 05315-970 São Paulo, SP, Brazil, \\ rafael@macbeth.if.usp.br \\ ** Universidade São Francisco, Centro de Ciências Exatas e Tecnológicas, \\ 13251-900 Itatiba, SP, Brazil \\ ****Instituto Nacional de Pesquisas Espaciais (INPE-LAS), C.P. 515, \\ 12201-970 São José do Campos, SP, Brazil \\ *****Universität Paderborn, FB-6 Physik, Warburger Strasse 100, D-33095 Paderborn, \\ Germany
}

Cite this article as: MRS internet J. Nitride Semicond. Res. 595, W3-40 (2000).

\begin{abstract}
Carrier concentration and mobility were measured for intrinsic cubic $\mathrm{InN}$ and $\mathrm{GaN}$, and for Si-doped cubic GaN as a function of temperature. Metallic n-type conductivity was found for the InN, while background p-type conductivity was observed for the intrinsic GaN layer. Doping the cubic GaN with Si two regimes were observed. For low Si-doping concentrations, the samples remain p-type. Increasing the Si-doping level, the background acceptors are compensated and the samples became highly degenerated n-type. From the carrier concentration dependence on temperature, the activation energy of the donor and acceptor levels was determined. Attempts were made to determine the scattering mechanisms responsible for the behavior of the mobility as a function of temperature.
\end{abstract}

\section{INTRODUCTION}

In the past few years nitride-based nanostructures have been successfully used in the fabrication of optoelectronic devices as well as in the development of high frequency and high-temperature electronic devices [1-4]. Most of the applications made so far are based on the hexagonal (h) phase of the nitride materials. However, cubic (c) GaN/GaAs (001) layers grown by metal organic chemical vapor deposition (MOCVD) and by plasma assisted molecular beam epitaxy (MBE) have recently been used to fabricate $p-n$ junction light emitting diodes [5-6]. In order to improve on the performance of these devices, further studies of the optical and electrical properties of the nitride layers are required. Particularly, the improvement of the $n$ - and p-type doping levels and carrier mobilities in these layers is a crucial task for the device technology, mainly for that involving the cubic phase of the materials $[7,8]$.

In the present work measurements of carrier concentrations and mobilities of unintentionally doped c-InN and c-GaN MBE grown epitaxial layers are performed as a function of temperature. $\mathrm{c}-\mathrm{GaN}$ samples doped with silicon are also investigated in the temperatures range 10 to $350 \mathrm{~K}$. The carrier concentrations and mobilities obtained for the 
cubic (zinc-blend) $\mathrm{InN}$ and $\mathrm{GaN}$ layers are compared with the results available for the corresponding hexagonal (wurtzite) nitride samples.

\section{SAMPLES}

The c-InN sample was grown on GaAs/InAs buffer layers firstly grown on GaAs (001) substrate by plasma-assisted MBE. We have used a Riber 32-system equipped with elemental sources of $\mathrm{Ga}$, As, In, Si and an Oxford Research CARS25 radio frequency plasma source for the reactive nitrogen. After the growth of the GaAs buffer layer at a temperature of $610^{\circ} \mathrm{C}$, we grew a $300 \mathrm{~nm}$ layer of InAs at a temperature of $480^{\circ} \mathrm{C}$ under $(2 \times 4)$ reconstruction. The growth of the $\mathrm{InN}$ layer, with about $300 \mathrm{~nm}$ width, was performed at a reduced temperature of $450^{\circ} \mathrm{C}$. Further details about the growth of the cInN layer are given in Refs. [9,10]. The same Riber 32-system was used to grow the intrinsic and the Si-doped c-GaN layers on GaAs (001) substrates. Elemental Si was evaporated at source temperatures between $750^{\circ} \mathrm{C}$ and $1100^{\circ} \mathrm{C}$.

The widths of the $\mathrm{c}-\mathrm{GaN}$ layers are of about $0.8 \mu \mathrm{m}[8,11]$. To perform the electrical characterization of the samples we have used Hall-effect measurements as a function of temperature at a $0.5 \mathrm{~T}$ magnetic field. Ohmic contacts using In were accomplished according to Van der Pauw geometry. The characteristics of our samples are shown in Table I.

Table I - Characteristics of the cubic nitride samples: $N_{D}$ is the nominal silicon concentration, $\mathrm{n}$ and $\mathrm{p}$ are the measured electron and hole concentrations at $300 \mathrm{~K}$, respectively.

\begin{tabular}{|c|c|c|c|}
\hline Sample & $\mathbf{N}_{\mathbf{D}}\left(\mathbf{c m}^{-3}\right)$ & $\mathbf{n}\left(\mathbf{c m}^{-\mathbf{3}}\right)$ & $\mathbf{p}\left(\mathbf{c m}^{-3}\right)$ \\
\hline c-InN & intrinsic & $5 \times 10^{20}$ & \\
\hline c-GaN & intrinsic & $1 \times 10^{16}$ \\
\hline \multicolumn{4}{|c|}{$\mathbf{c - G a N : S i}$} \\
\hline$\# 1$ & $3.7 \times 10^{17}$ & & $7.2 \times 10^{16}$ \\
\hline$\# 2$ & $1.2 \times 10^{19}$ & & $7.0 \times 10^{16}$ \\
\hline$\# 3$ & $1.2 \times 10^{19}$ & & \\
\hline$\# 4$ & $2.6 \times 10^{19}$ & $1.1 \times 10^{19}$ & \\
\hline$\# 5$ & $5.5 \times 10^{19}$ & $2.6 \times 10^{19}$ & \\
\hline$\# 6$ & $1.2 \times 10^{20}$ & $7.2 \times 10^{19}$ & \\
\hline$\# 7$ & $2.6 \times 10^{20}$ & $8.0 \times 10^{19}$ & \\
\hline$\# 8$ & $2.6 \times 10^{20}$ & $1.0 \times 10^{20}$ & \\
\hline$\# 9$ & $2.6 \times 10^{20}$ & $1.2 \times 10^{20}$ & \\
\hline
\end{tabular}

\section{INTRINSIC c-InN}

The measured carrier concentration in the unintentionally doped c-InN layer as a function of inverse temperature is shown in Fig.1. High-electron concentrations of the order of $10^{20} \mathrm{~cm}^{-3}$ were found in the range of temperature from 10 to $300 \mathrm{~K}$. This metallic behavior was also observed in MOCVD grown h-InN layers and attributed to the presence of native nitrogen vacancies in the sample [12]. The same behavior was also observed for 
metal organic MBE grown c-InN [13]. Fig. 1 shows that a sharp increase of the electron concentration occurs when the temperature raises above $200 \mathrm{~K}$. This behavior which was not observed in the h-InN layers may indicate that another deeper donor level, with activation energy of about $94,5 \mathrm{meV}$, exists in our c-InN layer. It is well known that ab initio calculation lead to zero or negative gap values for the InN binary compound. This fact has hindered severely our knowledge about native defects in this material from theory. On the other hand, very few attempts have been made so far to grow c-InN films [9]. Thus, systematic effort is required to identify the origin of the defects or impurities, which give rise to such high electron concentrations observed in our c-InN layer.

The electron mobilities measured by us in the c-InN layer as a function of temperature are shown in Fig.2. In the temperature range of 10 to $260 \mathrm{~K}$ the measured mobilities are one order of magnitude smaller than those observed for the h-InN films [12]. This probably indicates the best quality of the hexagonal films. Above $200 \mathrm{~K}$ a decrease of the mobilities follows the sharp increase in the concentrations. Attempts were made to adjust the observed behavior of the mobilities to different scattering and screening mechanisms. We expect that impurity scattering is involved in the changes of the mobility at lower temperatures and scattering by phonons is active at higher temperature, although the $\mathrm{T}$ dependencies obtained by us deviate from the expected ones for these mechanisms. Further investigations of both, h-InN and c-InN films are required to understand and control their electrical properties.

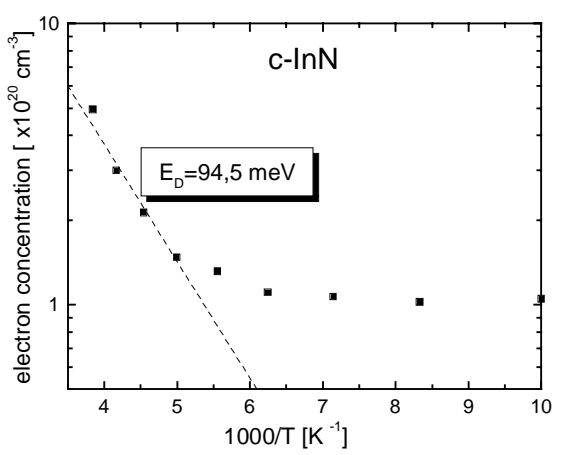

Figure 1: Electron concentration as a function of temperature for the intrinsic c-InN. The dashed line is used to calculate the activation energy $E_{D}=94.5 \mathrm{meV}$.

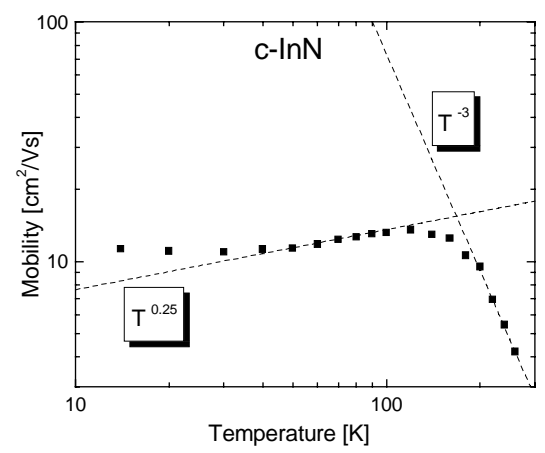

Figure 2: Mobility as a function of temperature for the intrinsic c-InN.

\section{INTRINSIC c-GaN}

The Hall carrier concentration measured as a function of temperature for the nominally undoped c-GaN sample is shown in Fig. 3. Due to the presence of an intrinsic acceptor level a background hole concentration is obtained. This concentration increases from $\mathrm{p}=3.7 \times 10^{13} \mathrm{~cm}^{-3}$ at $100 \mathrm{~K}$ (not shown in the Figure) to $\mathrm{p}=2.0 \times 10^{16} \mathrm{~cm}^{-3}$ for $\mathrm{T}=350 \mathrm{~K}$. The carrier concentration behavior near the room temperature region allows us to determine an activation energy of $\mathrm{E}_{\mathrm{A}}=166 \mathrm{meV}$ for the involved acceptor. Therefore, we 
can estimate an acceptor concentration of $\mathrm{N}_{\mathrm{A}} \approx 4 \times 10^{18} \mathrm{~cm}^{-3}$ for this nominally undoped sample. The measured hole mobility is presented in Fig. 4 as a function of temperature. The mobility initially increases with temperature reaching a maximum value of 1250 $\mathrm{cm}^{2} / \mathrm{Vs}$ at $\mathrm{T}=120 \mathrm{~K}$ and then decreases to a value of $283 \mathrm{~cm}^{2} / \mathrm{Vs}$ at room temperature. As can be seen in Fig. 4, the $\mathrm{T}^{3 / 2}$ behavior, shown at low temperature, suggest that in this region the mobility is limited by ionized impurity scattering. Above $120 \mathrm{~K}$ the decrease of mobility, proportional to $\mathrm{T}^{-3 / 2}$, is probably due to phonon scattering.

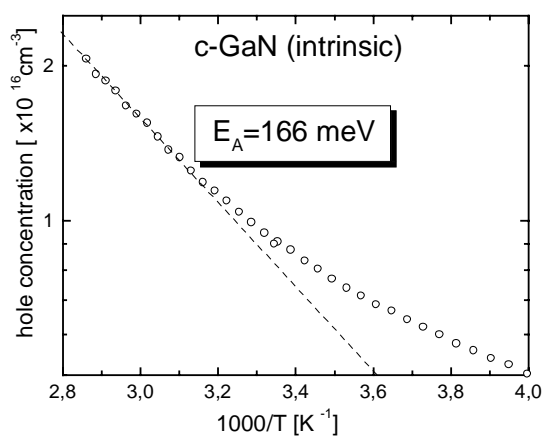

Figure 3: Hole concentration as a function of temperature for the intrinsic $\mathrm{c}-\mathrm{GaN}$. The dashed line is used to calculate the activation energy $\mathrm{E}_{\mathrm{A}}=166$ meV.

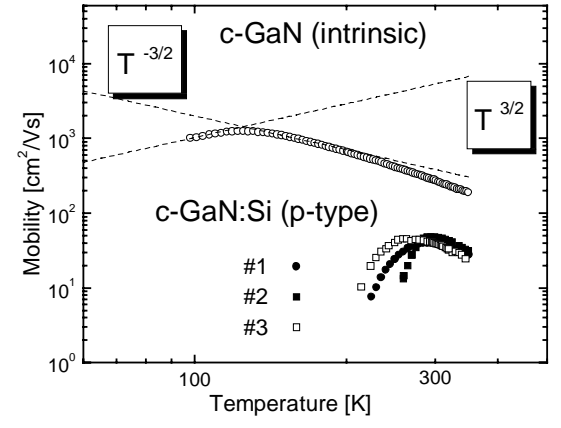

Figure 4: Mobility as a function of temperature for the intrinsic $\mathrm{c}-\mathrm{GaN}$ (opened circle) and for the lightly doped c-GaN:Si (closed circles, closed squares, and opened squares).

\section{LIGHTLY DOPED c-GaN:Si}

We expect to obtain n-type conductivity for c-GaN when it is doped with Si. Contrary to that, p-type conductivity is measured for samples with a Si concentration below $1.2 \mathrm{x}$ $10^{19} \mathrm{~cm}^{-3}$. This is due to the fact that the acceptor background, present in intrinsic c-GaN, is not yet completely compensated for these concentrations. The hole concentrations measured for the samples \#1, \#2, and \#3 are shown in Fig. 5. They all present the same behavior as a function of temperature, i.e., a decreasing of concentration as the temperature is lowered passing by a minimum and then increasing again when the temperature is further decreased. From the decrease of the concentration in the region near the room temperature an activation energy can be calculated yielding the values of 157,154 , and $171 \mathrm{meV}$ for the samples \#1, \#2, and \#3, respectively. All these values are very close to the activation energy measured for the acceptor level of the intrinsic c-GaN. This allows us to infer that the same acceptor level provides the measured high 
temperature hole concentrations in these samples. The increase of concentration at low temperature is probably due to a frozen of the carriers into a localized level. The shape of the mobility measured as a function of temperature (Fig. 4) is very similar to that presented by the intrinsic c-GaN. Nonetheless, the mobilities are much smaller and the $\mathrm{T}^{3 / 2}$ (low temperature) and $\mathrm{T}^{-3 / 2}$ (high temperature) dependence cannot describe their behavior anymore. This indicates that besides the scattering mechanisms described above, other mechanisms are involved.

\section{HEAVILY DOPED c-GaN:Si}

When the Si concentrations in c-GaN are higher than $2.5 \times 10^{19} \mathrm{~cm}^{-3}$ a n-type conductivity is obtained. For these concentrations the acceptor level is completely compensated and we have a degenerated semiconductor. The electron concentration as a function of temperature measured for samples \#4 to \#9 is shown in Fig. 6. A metallic behavior can be observed, since the electron concentration does not change as the temperature is varied, which is typical of highly degenerated semiconductors.

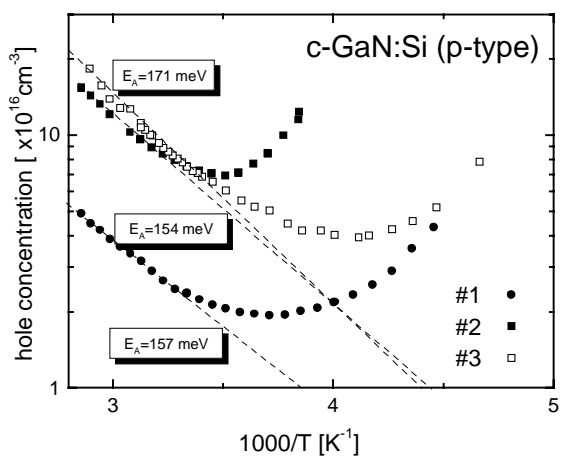

Figure 5: Hole concentration as a function of temperature for the lightly doped c-GaN:Si. The dashed lines are used to determine the activation energies.

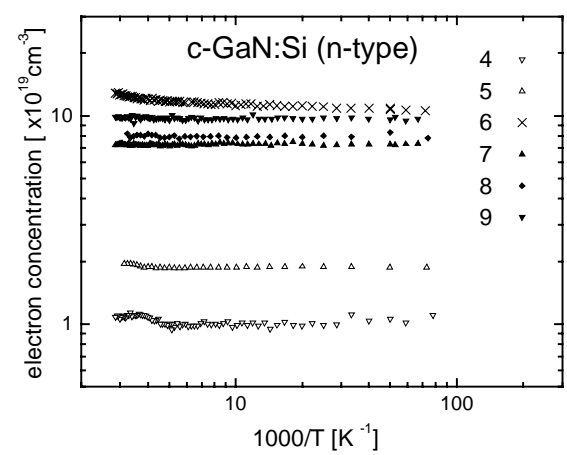

Figure 6: Electron concentration as a function of temperature for heavily doped c-GaN:Si.

\section{CONCLUSIONS}

Cubic InN and GaN binary semiconductor compounds were investigated by Hall effect measurements as a function of temperature. The InN sample shows a very high electron concentration $\left(\mathrm{n} \cong 10^{20} \mathrm{~cm}^{-3}\right)$. At temperatures above $200 \mathrm{~K}$, a donor with an activation energy of $E_{D}=94,5 \mathrm{meV}$ leads to a sharp increase of the electron concentration. The mobility at low temperature increases with $\mathrm{T}^{1 / 4}$ while a decrease with $\mathrm{T}^{-3}$ is observed at a high temperature region. Nominally undoped $\mathrm{c}-\mathrm{GaN}$ shows a p-type background concentration which varies from $10^{13}$ to $10^{16} \mathrm{~cm}^{-3}$ as the temperature is raised. The mobility dependence on temperature suggests that dislocations and ionized impurities are 
the scattering mechanisms at low temperature $\left(\mathrm{T}^{3 / 2}\right)$, while phonon scattering is the dominant one at a high temperature $\left(\mathrm{T}^{-3 / 2}\right)$. Si-doped c-GaN has p-type conductivity for Si-doping concentration below $\mathrm{N}_{\mathrm{D}} \cong 1.2 \times 10^{19} \mathrm{~cm}^{-3}$, and is highly n-type degenerated for $\mathrm{N}_{\mathrm{D}} \geq 1.2 \times 10^{19} \mathrm{~cm}^{-3}$. The behavior of the mobility as a function of temperature for the p-type $\mathrm{c}-\mathrm{GaN}: \mathrm{Si}$ is similar to that observed for the undoped sample, but its behavior is no longer described by $\mathrm{T}^{3 / 2}$ and $\mathrm{T}^{-3 / 2}$, indicating that other scattering mechanisms like dislocation scattering are taking place in our samples.

\section{ACKOWLEDGEMENTS}

This work was performed with partial support of a "CAPES/DAAD/PROBRAL" project within the Brazil/Germany scientific collaboration program. The authors also acknowledge financial support from FAPESP (Brazilian funding agency) and DFG (Deutsche Forschungsgemeinschaft).

\section{REFERENCES}

1. S. Nakamura and G. Fasol, The Blue Laser Diode (Springer, Berlin, 1997).

2. S. Nakamura, Semicond. Sci. Technol. 14, R27 (1999).

3. M.A. Khan, J.N. Kuznia, D.T. Olson, W.J. Schaff, J.W. Burm, and M. Shur, Appl. Phys. Lett. 65, 1121 (1995).

4. I.P. Smorchkova, C.R. Elsass, J.P. Ibbetson, R. Vetury, B. Heying, P. Fini, E. Haus, S.P. DenBaars, J.S. Speck, U.K. Mishra, J. Appl. Phys. 86, 4520 (1999).

5. H. Yang, L.X. Zheng, J.B. Li, X.J. Wang, D.P. Xu, Y.T. Wang, X.W. Hu, and P.D. Han, Appl. Phys. Lett. 74, 2498 (1999).

6. D.J. As, A. Richter, J. Busch, M. Lübbers, J. Mimkes, and K. Lischka, Appl. Phys. Lett. (in press).

7. L.K. Teles, L.M.R. Scolfaro, J.R. Leite, L.E. Ramos, A. Tabata, J.L.P. Castineira, and D.J. As, phys. stat. Sol. (b) 216, 541 (1999).

8. D.J. As, D. Schikora, A. Greiner, M. Lübbers, J. Mimkes, and K. Lischka, Phys. Rev. B54, R11 118 (1996).

9. A.P. Lima, A. Tabata, J.R. Leite, S. Kaiser, D. Schikora, B. Schöttker, T. Frey, D.J. As, and K. Lischka, J. Cryst. Growth 201/202, 396 (1999).

10. A. Tabata, A.P. Lima, L.K. Teles, L.M.R. Scolfaro, J.R. Leite, V. Lemos, B. Schöttker, T. Frey, D. Schikora, and K. Lischka, Appl. Phys. Lett. 74, 362 (1999).

11. D.J. As, A. Richter, J. Busch, B. Schöttker, M. Lübbers, J. Mimkes, D. Schikora, K. Lischka, W. Kriegseis, W. Burkhardt, and B.K. Meyer, MRS Symp. Proc. Vol. 595 (2000) paper W3. 81.

12. N. Miura, H. Ishii, A. Yamada, M. Konagai, Y. Yamauchi, and A. Yamamoto, Jpn. Appl. Phys. 36, L256 (1997).

13. W. Geerts, J.D. Mackenzie, C.R. Abernathy, S.J. Pearton, and T. Schmiedel, Solid State Electronics, 39, 1289 (1996).

F99W3.40 